\title{
Anisotropic Local Motions and Location of Amide Protons in Proteins
}

\author{
by Dimitri Bytchenkoff, Philippe Pelupessy and Geoffrey Bodenhausen
}

\section{SUPPORTING INFORMATION}

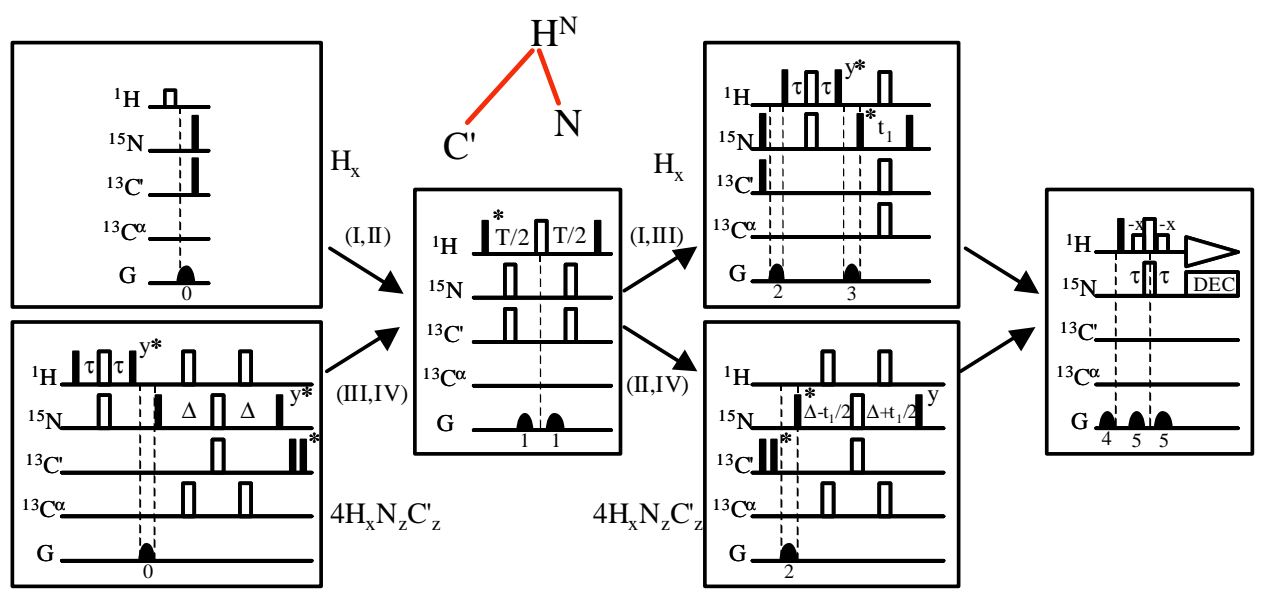

Figure S1a. Pulse sequences designed to measure the long-range dipole-dipole $R\left(C^{\prime} H^{N} / H^{N} N\right)$ cross-correlation rate. In experiments I and II, the operator $H_{X}^{N}$ is selected at the beginning of the relaxation delay $T$. In experiments III and IV, the coherence $4 H^{N}{ }_{X} N_{Z} C^{\prime}{ }_{Z}$ is initially excited. After the relaxation delay $T, H^{N}{ }_{X}$ is detected in experiments I and III and $4 H^{N}{ }_{X} N_{Z} C^{\prime}{ }_{Z}$ in experiments II and IV. Filled and open rectangles represent $90^{\circ}$ and $180^{\circ}$ pulses respectively. The pulses marked with an asterisk are phase-alternated independently in concert with the receiver. The delay $\tau$ was set to $2.65 \mathrm{~ms} \approx 1 /\left[4^{1} \mathrm{~J}\left(\mathrm{H}^{\mathrm{N}} \mathrm{N}\right)\right]$ and the delay $\Delta$ to $16.5 \mathrm{~ms} \approx 1 /\left[4^{1} \mathrm{~J}\left(\mathrm{NC}^{\prime}\right)\right]$.

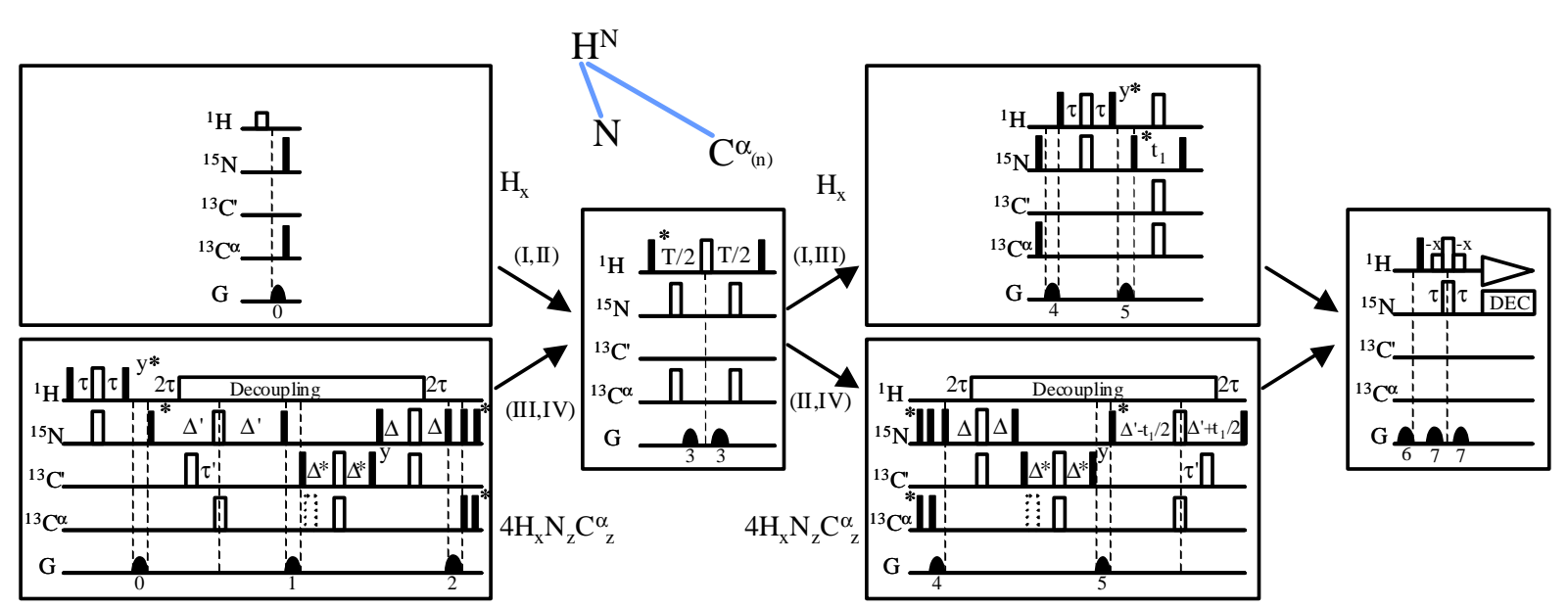

Figure S1b. Pulse sequences designed to measure the long-range dipole-dipole $R\left(N H^{N} / H^{N} C^{\alpha}\right)$ cross-correlation rate. In experiments I and II, the operator $H^{N}{ }_{X}$ is selected at the beginning of the relaxation delay $T$. In experiments III and IV, the coherence $4 H^{N}{ }_{X} N_{Z} C^{\alpha}{ }_{Z}$ is initially excited. After the relaxation delay $T, H^{N}{ }_{X}$ is detected in experiments I and III and $4 H^{N}{ }_{X} N_{Z} C^{\alpha}{ }_{Z}$ in experiments II and IV. The values of the delays $\tau, \Delta, \Delta^{*}, \Delta^{\prime}$ and $\tau^{\prime}$ are set to $2.65 \mathrm{~ms} \approx$ $1 /\left[4^{1} \mathrm{~J}\left(\mathrm{H}^{\mathrm{N}} \mathrm{N}\right)\right], 16.5 \mathrm{~ms} \approx 1 /\left[4^{1} \mathrm{~J}\left(\mathrm{NC}^{\prime}\right)\right], 4.5 \mathrm{~ms} \approx 1 /\left[4^{1} \mathrm{~J}\left(\mathrm{C}^{\prime} \mathrm{C}^{\alpha}\right)\right], 26 \mathrm{~ms}$ and $4.75 \mathrm{~ms} \approx 0.5\left(\Delta^{\prime}-\Delta\right)$, respectively. The delay $\Delta$ and $\tau^{\prime}$ are adjusted for the transfer $2 N_{X} H^{N}{ }_{Z} \rightarrow 8 C^{\alpha}{ }_{Z}(n-1) C^{\prime}{ }_{Z} N_{X} C^{\alpha}{ }_{Z}(n)$. The dashed pulses compensate for Bloch-Siegert effects. 


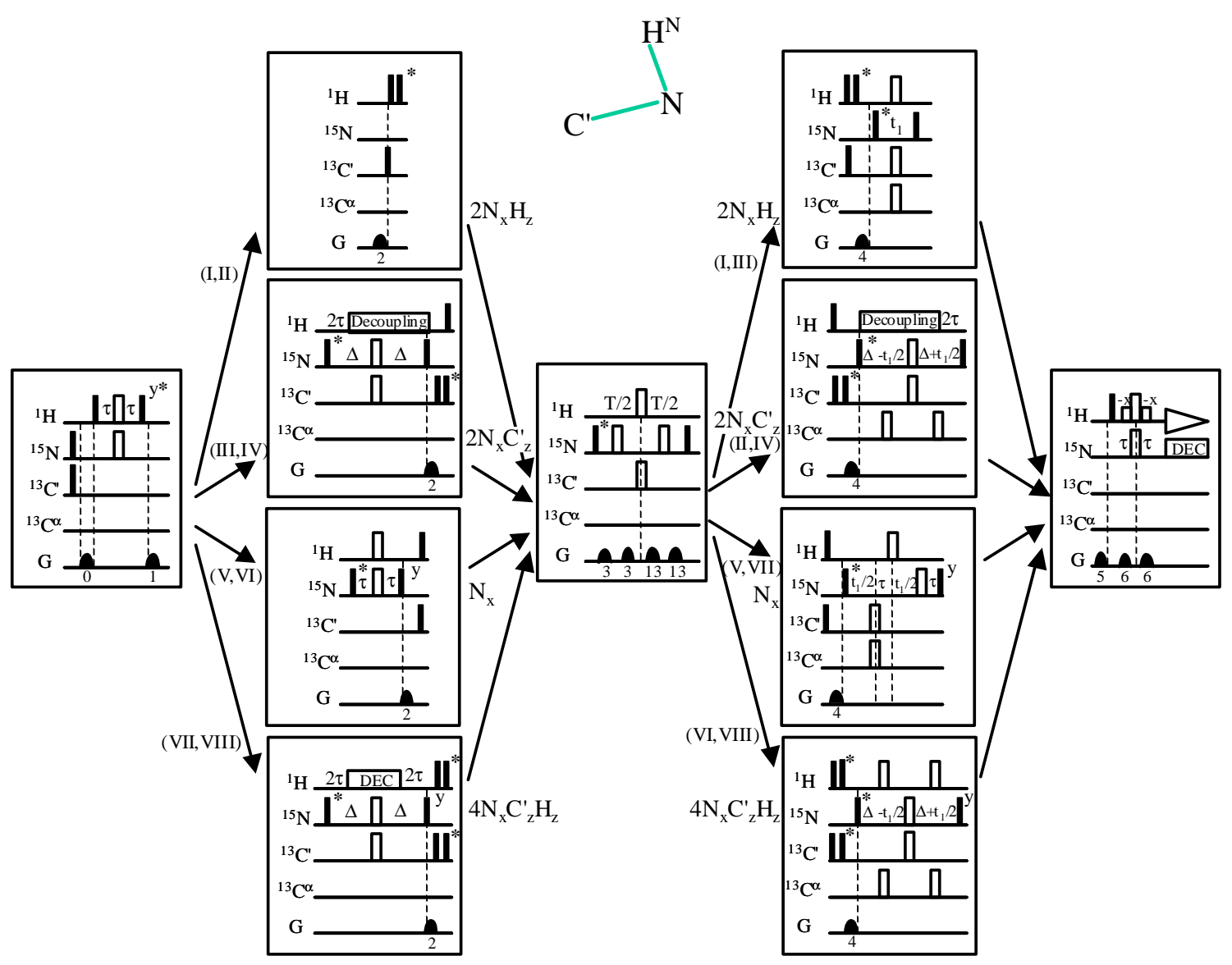

Figure S1c. Pulse sequences designed to measure the short-range dipole-dipole $R\left(H^{N} N / N C^{\prime}\right)$ cross-correlation rate. Before the relaxation delay $T$, the operator $2 N_{x} H_{z}^{N}$, is prepared in experiments I and II, $2 N_{X} C^{\prime}{ }_{Z}$ in III and IV, $N_{X}$ in V and VI, and $4 N_{X} H^{N}{ }_{Z} C^{\prime}{ }_{Z}$ in VII and VIII. After the relaxation delay T, the coherence $2 N_{X} H^{N}{ }_{Z}$ is detected in experiments I and III, $2 N_{X} C^{\prime}{ }_{Z}$ in II and IV, $N_{X}$ in V and VI, $4 N_{X} H^{N}{ }_{Z} C^{\prime}{ }_{Z}$ in VI and VIII. The delay $\tau$ was set to $2.65 \mathrm{~ms}$ $\approx 1 /\left[4^{1} \mathrm{~J}\left(\mathrm{H}^{\mathrm{N}} \mathrm{N}\right)\right]$ and the delay $\Delta$ to $16.5 \mathrm{~ms} \approx 1 /\left[4^{1} \mathrm{~J}\left(\mathrm{NC}^{\prime}\right)\right]$. 
Anisotropic Local Motions and Location of Amide Protons in Proteins

\begin{tabular}{|c|c|c|c|c|c|c|}
\hline Residue & $R\left(N H^{N} / H^{N} C^{\prime}\right)$ & $+/-$ & $R\left(\mathrm{NH}^{\mathrm{N}} / \mathrm{HNC}^{\alpha}\right)$ & $+/-$ & $R\left(H^{N} N / N C^{\prime}\right)$ & $+/-$ \\
\hline 2 & 1.11 & 0.01 & 0.96 & 0.01 & 0.21 & 0.01 \\
\hline 3 & 1.17 & 0.03 & 0.86 & 0.02 & 0.20 & 0.01 \\
\hline 4 & 1.18 & 0.02 & 0.93 & 0.02 & 0.19 & 0.01 \\
\hline 5 & 1.14 & 0.03 & 0.90 & 0.03 & 0.24 & 0.02 \\
\hline 6 & 1.15 & 0.02 & 0.92 & 0.01 & 0.18 & 0.01 \\
\hline 7 & 1.10 & 0.02 & 0.88 & 0.01 & 0.21 & 0.01 \\
\hline 8 & 0.93 & 0.02 & 0.72 & 0.01 & 0.16 & 0.01 \\
\hline 9 & 0.86 & 0.03 & 0.68 & 0.03 & 0.17 & 0.01 \\
\hline 10 & 0.96 & 0.02 & 0.68 & 0.03 & 0.18 & 0.01 \\
\hline 11 & 0.76 & 0.02 & 0.71 & 0.02 & 0.20 & 0.01 \\
\hline 12 & 0.96 & 0.01 & 0.73 & 0.01 & 0.21 & 0.01 \\
\hline 13 & 1.17 & 0.02 & 0.91 & 0.02 & 0.17 & 0.01 \\
\hline 14 & 1.02 & 0.01 & 0.85 & 0.01 & 0.22 & 0.01 \\
\hline 15 & 1.22 & 0.03 & 0.91 & 0.02 & 0.16 & 0.01 \\
\hline 16 & 1.01 & 0.01 & 0.90 & 0.01 & 0.22 & 0.01 \\
\hline 17 & 1.23 & 0.02 & 0.93 & 0.01 & 0.16 & 0.01 \\
\hline 18 & 1.15 & 0.02 & 0.96 & 0.02 & 0.20 & 0.01 \\
\hline 20 & 1.16 & 0.02 & 0.91 & 0.02 & 0.17 & 0.01 \\
\hline 22 & 1.12 & 0.02 & 0.88 & 0.02 & 0.18 & 0.01 \\
\hline 23 & 1.19 & 0.03 & 0.89 & 0.03 & 0.17 & 0.01 \\
\hline 25 & 1.23 & 0.03 & 0.90 & 0.03 & 0.14 & 0.01 \\
\hline 26 & 1.20 & 0.03 & 0.95 & 0.05 & 0.14 & 0.01 \\
\hline 27 & 1.22 & 0.03 & 0.95 & 0.04 & 0.18 & 0.02 \\
\hline 29 & 1.22 & 0.02 & 0.90 & 0.02 & 0.15 & 0.01 \\
\hline 30 & 1.17 & 0.04 & 1.01 & 0.05 & 0.17 & 0.02 \\
\hline 32 & 1.31 & 0.02 & 0.94 & 0.02 & 0.15 & 0.01 \\
\hline 33 & 1.12 & 0.02 & 0.92 & 0.02 & 0.15 & 0.01 \\
\hline 34 & 1.10 & 0.03 & 0.90 & 0.02 & 0.22 & 0.01 \\
\hline 35 & 1.21 & 0.03 & 0.98 & 0.04 & 0.16 & 0.01 \\
\hline 36 & 0.92 & 0.02 & 0.79 & 0.02 & 0.26 & 0.01 \\
\hline 39 & 1.15 & 0.02 & 0.83 & 0.02 & 0.15 & 0.01 \\
\hline 40 & 1.20 & 0.03 & 0.98 & 0.02 & 0.17 & 0.01 \\
\hline 41 & 1.10 & 0.03 & 0.76 & 0.02 & 0.20 & 0.01 \\
\hline 42 & 1.22 & 0.02 & 0.95 & 0.01 & 0.13 & 0.01 \\
\hline 43 & 1.13 & 0.02 & 0.83 & 0.02 & 0.21 & 0.01 \\
\hline 44 & 1.17 & 0.03 & 0.97 & 0.02 & 0.17 & 0.01 \\
\hline 45 & 1.15 & 0.02 & 0.93 & 0.02 & 0.20 & 0.01 \\
\hline 46 & 1.20 & 0.03 & 0.85 & 0.02 & 0.10 & 0.01 \\
\hline 47 & 1.01 & 0.02 & 0.75 & 0.02 & 0.18 & 0.01 \\
\hline 48 & 1.17 & 0.02 & 0.87 & 0.02 & 0.18 & 0.01 \\
\hline 49 & 1.07 & 0.01 & 0.79 & 0.01 & 0.17 & 0.01 \\
\hline 50 & 1.11 & 0.02 & 0.90 & 0.01 & 0.18 & 0.01 \\
\hline 51 & 1.15 & 0.03 & 0.96 & 0.02 & 0.19 & 0.01 \\
\hline 52 & 1.15 & 0.02 & 0.90 & 0.01 & 0.19 & 0.01 \\
\hline 54 & 0.97 & 0.02 & 0.76 & 0.03 & 0.24 & 0.01 \\
\hline 55 & 1.09 & 0.03 & 0.84 & 0.03 & 0.18 & 0.01 \\
\hline 56 & 1.21 & 0.02 & 0.99 & 0.02 & 0.12 & 0.01 \\
\hline 57 & 1.15 & 0.02 & 0.77 & 0.03 & 0.17 & 0.01 \\
\hline 58 & 1.18 & 0.03 & 0.83 & 0.03 & 0.14 & 0.01 \\
\hline 59 & 1.14 & 0.03 & 0.89 & 0.02 & 0.16 & 0.01 \\
\hline 60 & 1.13 & 0.02 & 0.84 & 0.02 & 0.19 & 0.01 \\
\hline 61 & 1.20 & 0.02 & 0.86 & 0.02 & 0.13 & 0.01 \\
\hline 62 & 0.84 & 0.02 & 0.73 & 0.01 & 0.23 & 0.01 \\
\hline 63 & 1.28 & 0.01 & 0.89 & 0.01 & 0.15 & 0.01 \\
\hline 64 & 1.24 & 0.02 & 0.89 & 0.02 & 0.15 & 0.01 \\
\hline 65 & 1.08 & 0.03 & 0.70 & 0.03 & 0.16 & 0.01 \\
\hline 66 & 1.07 & 0.02 & 0.89 & 0.02 & 0.22 & 0.01 \\
\hline 67 & 1.18 & 0.02 & 0.86 & 0.02 & 0.21 & 0.01 \\
\hline 68 & 1.11 & 0.03 & 0.90 & 0.02 & 0.21 & 0.01 \\
\hline 70 & 1.18 & 0.02 & 0.90 & 0.02 & 0.22 & 0.01 \\
\hline 71 & 0.99 & 0.01 & 0.78 & 0.01 & 0.19 & 0.01 \\
\hline 74 & 0.41 & 0.01 & 0.28 & 0.00 & 0.11 & 0.00 \\
\hline 75 & 0.30 & 0.01 & 0.14 & 0.01 & 0.01 & 0.01 \\
\hline 76 & 0.15 & 0.00 & 0.07 & 0.00 & 0.01 & 0.01 \\
\hline
\end{tabular}

Table S1. Experimental cross-correlation rates $R\left(C^{\prime} H^{N} / H^{N} N\right), R\left(N H^{N} / H^{N} C^{\alpha}\right)$ and $R\left(H^{N} N / N C^{\prime}\right)$ measured in ${ }^{15} \mathrm{~N}$ - and ${ }^{13} \mathrm{C}$-labelled human ubiquitin at $300 \mathrm{~K}$, using a DMX-600 NMR Bruker spectrometer equipped with a triple-axes pulsed field gradient TBI probe optimised for ${ }^{1} \mathrm{H}$ detection. For $R\left(C^{\prime} H^{N} / H^{N} N\right) 58$ complex points were acquired in the indirect dimension, the recovery delay was $1.3 \mathrm{~s}$, the number of scans was 64 , resulting in a total experimental time of around 12 hours. The experiment was performed twice with a relaxation delay $T$ of 50 and 60 ms. For $R\left(N H^{N} / H^{N} C^{\alpha}\right): 72$ complex points, a recovery delay of $1.3 \mathrm{~s}$ with 256 scans per increment, experimental time 60 hours, relaxation delay $T=60 \mathrm{~ms}$. For $R\left(H^{N} N / N C^{\prime}\right)$ : 58 complex points, a recovery delay of $1.3 \mathrm{~s}$ with $128 \mathrm{scans}$ per increment, experimental time 48 hours, relaxation delay $T=60$ and $100 \mathrm{~ms}$. The error was estimated from the noise level and evaluated by comparing the results at different relaxation delays. 
Anisotropic Local Motions and Location of Amide Protons in Proteins

\begin{tabular}{|c|c|c|c|c|c|c|c|c|}
\hline Residue & $\alpha\left[^{\circ}\right]$ & $+/-$ & $\sigma_{\gamma}[\stackrel{0}{]}]$ & $+/-$ & $\sigma_{\alpha}=\sigma_{\beta}[\stackrel{\circ}{-}]$ & $+/-$ & $\tau_{\text {int }}[p s]$ & $+/-$ \\
\hline 2 & -1.43 & 0.66 & 13.67 & 0.37 & 6.63 & 1.91 & 9.14 & 3.46 \\
\hline 3 & 1.21 & 2.22 & 10.72 & 1.50 & 8.31 & 3.74 & 4.31 & 6.57 \\
\hline 4 & -1.53 & 0.54 & 11.85 & 0.48 & 0.86 & 1.92 & -0.44 & 7.40 \\
\hline 5 & -0.53 & 1.29 & 11.13 & 1.03 & 2.05 & 3.31 & 3.04 & 10.90 \\
\hline 6 & -1.37 & 0.52 & 12.85 & 0.39 & 1.74 & 2.42 & 38.95 & 4.88 \\
\hline 7 & -2.64 & 0.44 & 12.60 & 0.37 & 1.88 & 2.31 & 48.47 & 5.36 \\
\hline 8 & 4.50 & 1.53 & 12.72 & 0.57 & 14.96 & 0.94 & 63.20 & 3.12 \\
\hline 9 & -2.83 & 0.99 & 18.25 & 0.48 & 5.94 & 2.98 & 38.99 & 2.35 \\
\hline 10 & -3.91 & 0.85 & 15.51 & 0.57 & 5.69 & 2.67 & 69.79 & 4.18 \\
\hline 11 & 0.53 & 1.26 & 18.22 & 0.42 & 12.18 & 1.08 & 35.85 & 1.55 \\
\hline 12 & -1.59 & 0.62 & 15.01 & 0.33 & 7.01 & 1.53 & 18.55 & 2.48 \\
\hline 13 & -1.73 & 0.35 & 13.54 & 0.32 & 0.17 & 0.86 & 50.59 & 4.80 \\
\hline 14 & -1.38 & 0.67 & 13.70 & 0.42 & 5.42 & 2.32 & 29.22 & 3.42 \\
\hline 15 & -2.10 & 0.55 & 12.94 & 0.47 & 0.66 & 1.75 & 24.59 & 7.22 \\
\hline 16 & -1.45 & 0.46 & 14.67 & 0.25 & 6.85 & 1.23 & 58.33 & 3.00 \\
\hline 17 & -1.47 & 0.75 & 12.71 & 0.49 & 4.58 & 2.79 & -0.59 & 4.52 \\
\hline 18 & -2.18 & 0.75 & 13.63 & 0.48 & 4.81 & 2.85 & 3.27 & 4.63 \\
\hline 20 & -2.55 & 0.60 & 13.78 & 0.46 & 1.93 & 2.58 & 23.35 & 4.53 \\
\hline 22 & 2.00 & 2.03 & 9.14 & 1.49 & 10.89 & 2.33 & 3.79 & 5.22 \\
\hline 23 & -2.07 & 0.73 & 12.07 & 0.67 & 0.97 & 2.23 & 14.44 & 7.02 \\
\hline 25 & 2.69 & 1.51 & 7.17 & 1.35 & 12.50 & 1.63 & 11.80 & 4.68 \\
\hline 26 & 0.29 & 2.66 & 10.58 & 2.17 & 6.73 & 4.85 & 29.81 & 5.99 \\
\hline 27 & -0.32 & 2.15 & 6.38 & 3.29 & 6.59 & 4.84 & -4.82 & 19.19 \\
\hline 29 & 2.19 & 2.00 & 7.65 & 1.68 & 11.34 & 2.44 & 23.16 & 4.62 \\
\hline 30 & 2.99 & 2.55 & 8.17 & 2.19 & 9.63 & 3.95 & -5.23 & 9.99 \\
\hline 32 & 0.64 & 1.91 & 7.97 & 1.84 & 8.82 & 3.25 & -2.34 & 8.20 \\
\hline 33 & 2.40 & 2.31 & 10.97 & 1.38 & 10.71 & 2.63 & 29.94 & 3.62 \\
\hline 34 & -1.09 & 0.90 & 12.58 & 0.65 & 2.50 & 3.01 & 16.02 & 5.17 \\
\hline 35 & -2.61 & 1.92 & 11.08 & 1.64 & 5.35 & 4.42 & 26.42 & 5.50 \\
\hline 36 & -1.27 & 0.71 & 18.50 & 0.34 & 4.43 & 2.61 & 20.39 & 1.88 \\
\hline 39 & 0.39 & 1.84 & 10.08 & 1.32 & 10.62 & 2.15 & 19.84 & 3.72 \\
\hline 40 & -0.26 & 1.52 & 11.61 & 1.06 & 4.61 & 3.77 & 39.68 & 6.39 \\
\hline 41 & -3.19 & 0.44 & 13.82 & 0.40 & 0.53 & 1.51 & 39.46 & 5.00 \\
\hline 42 & -1.14 & 1.27 & 10.58 & 0.97 & 6.30 & 3.17 & 20.06 & 5.43 \\
\hline 43 & -2.77 & 0.33 & 12.72 & 0.34 & 0.24 & 0.99 & 36.17 & 5.78 \\
\hline 44 & 1.05 & 1.97 & 10.55 & 1.42 & 7.16 & 3.80 & 28.94 & 5.52 \\
\hline 45 & -1.59 & 1.07 & 10.80 & 0.87 & 3.81 & 3.45 & 15.55 & 6.57 \\
\hline 46 & -3.41 & 0.79 & 12.77 & 0.59 & 4.07 & 3.26 & 60.34 & 7.03 \\
\hline 47 & -0.69 & 1.79 & 13.04 & 1.03 & 9.98 & 2.10 & 44.34 & 3.44 \\
\hline 48 & -2.73 & 0.37 & 14.76 & 0.28 & 1.08 & 1.81 & 1.80 & 3.65 \\
\hline 49 & -0.91 & 0.59 & 13.59 & 0.32 & 8.63 & 1.08 & 14.53 & 2.53 \\
\hline 50 & -1.51 & 0.66 & 13.57 & 0.44 & 3.06 & 2.86 & 28.28 & 4.29 \\
\hline 51 & -0.54 & 1.82 & 13.22 & 1.00 & 6.34 & 3.82 & 36.05 & 4.56 \\
\hline 52 & 2.37 & 1.89 & 12.74 & 0.94 & 11.23 & 1.77 & 39.95 & 3.03 \\
\hline 54 & -3.58 & 0.39 & 16.66 & 0.32 & 0.00 & 0.00 & 18.09 & 2.93 \\
\hline 55 & 3.47 & 2.05 & 9.96 & 1.21 & 12.37 & 2.01 & 34.34 & 4.34 \\
\hline 56 & 3.12 & 1.10 & 6.02 & 1.00 & 12.52 & 1.16 & 28.36 & 4.44 \\
\hline 57 & -3.77 & 0.39 & 13.60 & 0.43 & 0.01 & 0.14 & 38.91 & 5.31 \\
\hline 58 & -0.63 & 2.23 & 11.37 & 1.50 & 9.03 & 3.51 & 7.19 & 5.05 \\
\hline 59 & -1.51 & 0.82 & 13.60 & 0.58 & 2.18 & 2.87 & 14.52 & 4.80 \\
\hline 60 & -1.50 & 1.45 & 11.40 & 1.07 & 5.73 & 3.61 & 45.47 & 6.51 \\
\hline 61 & -2.61 & 0.85 & 12.93 & 0.63 & 3.40 & 3.14 & 10.39 & 5.19 \\
\hline 62 & -2.12 & 0.58 & 17.14 & 0.28 & 6.14 & 1.95 & 26.93 & 2.17 \\
\hline 63 & -2.37 & 0.49 & 12.95 & 0.35 & 4.36 & 2.33 & 59.46 & 5.02 \\
\hline 64 & -2.37 & 0.50 & 11.87 & 0.43 & 1.47 & 2.34 & 4.95 & 6.27 \\
\hline 65 & -4.50 & 0.45 & 15.61 & 0.42 & 0.00 & 0.00 & 5.35 & 4.42 \\
\hline 66 & -1.47 & 0.54 & 13.21 & 0.39 & 2.24 & 2.48 & 28.52 & 4.37 \\
\hline 67 & -1.96 & 0.33 & 12.53 & 0.35 & 0.00 & 0.00 & 39.70 & 5.22 \\
\hline 68 & 0.51 & 1.94 & 10.37 & 1.40 & 7.29 & 3.75 & -1.05 & 6.60 \\
\hline 70 & -1.96 & 0.37 & 11.58 & 0.41 & 0.06 & 0.48 & 20.65 & 6.79 \\
\hline 71 & 0.09 & 0.78 & 13.36 & 0.42 & 10.24 & 1.02 & 72.17 & 3.73 \\
\hline 74 & -6.18 & 0.24 & 21.83 & 0.08 & 15.59 & 0.28 & 88.36 & 1.01 \\
\hline 75 & -12.77 & 0.56 & 22.57 & 0.14 & 19.33 & 0.31 & 91.40 & 0.85 \\
\hline 76 & 19.87 & 23.62 & 23.09 & 0.37 & 23.29 & 0.22 & 53.94 & 0.28 \\
\hline
\end{tabular}

Table S2. The amplitudes $\sigma_{\gamma}$ and $\sigma_{\alpha}=\sigma_{\beta}$ of the axially-symmetric 3-D GAF model as well as the angles $\alpha$ that determine the location of the amide $\mathrm{H}^{\mathrm{N}}$ proton and the internal correlation time $\tau_{\text {int }}$, determined from the rates of Table S1 using an axially-symmetric 3-D GAF model. 
Anisotropic Local Motions and Location of Amide Protons in Proteins

\begin{tabular}{|c|c|c|c|c|c|c|c|c|}
\hline Residue & $\mathrm{C}^{\prime} \mathrm{NC}^{\alpha}\left[{ }^{\circ}\right]$ & $+/-$ & C $\mathrm{NH}^{\mathrm{N}}[\stackrel{\circ}{\mathrm{a}}]$ & $+/-$ & $\left.\sigma_{\gamma} \stackrel{\circ}{ }\right]^{\prime}\left(=2 \sigma_{\alpha}=2 \sigma_{\beta}\right)$ & $+/-$ & $\tau_{\text {int }}[p s]$ & $+/-$ \\
\hline 2 & 121.56 & 0.97 & 120.66 & 0.33 & 13.70 & 0.32 & 8.69 & 3.96 \\
\hline 3 & 120.11 & 1.96 & 120.09 & 0.61 & 12.30 & 0.65 & 2.25 & 7.48 \\
\hline 4 & 123.78 & 1.56 & 119.47 & 0.54 & 10.56 & 0.70 & -2.25 & 9.34 \\
\hline 5 & 123.12 & 2.15 & 119.01 & 0.70 & 10.29 & 0.99 & 0.23 & 13.81 \\
\hline 6 & 123.19 & 1.25 & 119.41 & 0.42 & 11.83 & 0.49 & 37.94 & 5.40 \\
\hline 7 & 123.05 & 1.10 & 120.82 & 0.35 & 11.65 & 0.44 & 47.10 & 5.83 \\
\hline 8 & 114.93 & 1.73 & 122.91 & 0.49 & 16.96 & 0.36 & 68.64 & 3.42 \\
\hline 9 & 123.37 & 2.04 & 120.69 & 0.60 & 17.48 & 0.47 & 38.38 & 2.47 \\
\hline 10 & 122.35 & 1.50 & 122.51 & 0.37 & 15.07 & 0.34 & 68.55 & 3.71 \\
\hline 11 & 118.02 & 1.62 & 121.63 & 0.45 & 19.48 & 0.26 & 35.95 & 1.76 \\
\hline 12 & 121.76 & 0.96 & 120.62 & 0.31 & 14.91 & 0.27 & 18.21 & 2.87 \\
\hline 13 & 124.93 & 1.31 & 119.08 & 0.45 & 11.88 & 0.52 & 50.77 & 5.81 \\
\hline 14 & 122.02 & 1.01 & 120.18 & 0.32 & 13.41 & 0.31 & 28.60 & 3.86 \\
\hline 15 & 124.40 & 1.73 & 119.75 & 0.60 & 11.47 & 0.72 & 23.53 & 8.50 \\
\hline 16 & 121.81 & 0.73 & 120.45 & 0.25 & 14.56 & 0.22 & 57.71 & 3.02 \\
\hline 17 & 122.06 & 1.15 & 120.28 & 0.36 & 12.39 & 0.42 & -0.85 & 4.97 \\
\hline 18 & 122.19 & 1.30 & 120.89 & 0.44 & 13.24 & 0.44 & 2.92 & 5.21 \\
\hline 20 & 123.51 & 1.53 & 120.48 & 0.49 & 12.71 & 0.52 & 22.64 & 5.11 \\
\hline 22 & 118.96 & 1.43 & 121.03 & 0.40 & 12.25 & 0.45 & 0.22 & 6.43 \\
\hline 23 & 124.04 & 1.99 & 120.06 & 0.60 & 10.79 & 0.83 & 13.05 & 8.44 \\
\hline 25 & 116.30 & 2.48 & 122.07 & 0.64 & 12.20 & 0.69 & 8.89 & 6.62 \\
\hline 26 & 120.45 & 2.90 & 120.45 & 0.50 & 11.86 & 0.62 & 29.41 & 6.95 \\
\hline 27 & 120.15 & 2.59 & 121.34 & 0.65 & 9.09 & 1.08 & -10.04 & 19.62 \\
\hline 29 & 118.24 & 1.85 & 121.42 & 0.50 & 11.73 & 0.62 & 22.72 & 6.34 \\
\hline 30 & 117.79 & 3.68 & 119.90 & 0.70 & 11.44 & 0.92 & -10.36 & 11.53 \\
\hline 32 & 119.82 & 1.48 & 121.04 & 0.42 & 10.53 & 0.59 & -6.53 & 8.81 \\
\hline 33 & 119.03 & 1.74 & 120.42 & 0.45 & 13.46 & 0.51 & 30.33 & 4.38 \\
\hline 34 & 122.97 & 1.71 & 119.40 & 0.48 & 11.76 & 0.65 & 15.26 & 5.93 \\
\hline 35 & 121.32 & 2.45 & 122.38 & 0.58 & 11.55 & 0.79 & 25.73 & 6.28 \\
\hline 36 & 124.53 & 1.51 & 118.16 & 0.47 & 17.31 & 0.34 & 20.14 & 2.14 \\
\hline 39 & 119.04 & 1.33 & 121.99 & 0.37 & 12.67 & 0.40 & 19.06 & 4.83 \\
\hline 40 & 121.80 & 1.70 & 119.53 & 0.48 & 11.61 & 0.64 & 38.94 & 6.79 \\
\hline 41 & 124.62 & 1.52 & 120.73 & 0.50 & 12.29 & 0.60 & 39.03 & 5.84 \\
\hline 42 & 121.04 & 1.35 & 121.04 & 0.45 & 11.21 & 0.56 & 19.19 & 6.40 \\
\hline 43 & 124.57 & 1.28 & 120.42 & 0.42 & 11.08 & 0.61 & 36.07 & 6.98 \\
\hline 44 & 120.67 & 1.77 & 119.58 & 0.57 & 11.68 & 0.65 & 28.46 & 6.32 \\
\hline 45 & 121.99 & 1.54 & 120.66 & 0.49 & 10.61 & 0.69 & 14.46 & 7.63 \\
\hline 46 & 122.27 & 1.57 & 122.19 & 0.49 & 12.33 & 0.63 & 59.29 & 7.07 \\
\hline 47 & 119.52 & 1.52 & 122.04 & 0.35 & 14.50 & 0.34 & 45.27 & 3.58 \\
\hline 48 & 124.15 & 1.13 & 120.20 & 0.36 & 13.46 & 0.35 & 1.84 & 4.19 \\
\hline 49 & 120.57 & 0.76 & 121.09 & 0.26 & 14.28 & 0.23 & 13.98 & 3.02 \\
\hline 50 & 122.86 & 1.35 & 119.71 & 0.47 & 12.78 & 0.48 & 27.49 & 4.77 \\
\hline 51 & 121.34 & 2.06 & 120.22 & 0.57 & 13.47 & 0.69 & 35.60 & 4.82 \\
\hline 52 & 118.93 & 1.24 & 120.38 & 0.38 & 14.87 & 0.32 & 41.14 & 3.36 \\
\hline 54 & 128.12 & 1.45 & 119.75 & 0.52 & 14.49 & 0.45 & 17.47 & 3.59 \\
\hline 55 & 117.04 & 2.35 & 121.16 & 0.60 & 13.77 & 0.62 & 35.57 & 5.37 \\
\hline 56 & 116.94 & 1.75 & 121.72 & 0.50 & 11.67 & 0.59 & 29.31 & 6.37 \\
\hline 57 & 126.64 & 1.49 & 121.02 & 0.47 & 11.60 & 0.61 & 39.67 & 6.63 \\
\hline 58 & 119.67 & 2.06 & 122.01 & 0.52 & 13.02 & 0.60 & 5.53 & 5.77 \\
\hline 59 & 123.44 & 1.81 & 119.51 & 0.50 & 12.57 & 0.65 & 13.86 & 5.51 \\
\hline 60 & 121.30 & 1.63 & 121.15 & 0.48 & 11.75 & 0.61 & 45.05 & 6.53 \\
\hline 61 & 122.63 & 1.65 & 121.13 & 0.49 & 12.32 & 0.57 & 9.76 & 5.94 \\
\hline 62 & 122.98 & 1.12 & 120.19 & 0.38 & 16.49 & 0.29 & 26.51 & 2.45 \\
\hline 63 & 122.24 & 0.87 & 121.03 & 0.31 & 12.50 & 0.32 & 57.92 & 4.98 \\
\hline 64 & 123.17 & 1.32 & 120.57 & 0.45 & 10.86 & 0.59 & 4.03 & 7.42 \\
\hline 65 & 128.37 & 1.64 & 121.00 & 0.56 & 13.28 & 0.61 & 3.06 & 5.92 \\
\hline 66 & 123.06 & 1.16 & 119.55 & 0.34 & 12.29 & 0.41 & 27.69 & 4.87 \\
\hline 67 & 126.42 & 1.23 & 119.12 & 0.44 & 10.22 & 0.64 & 42.38 & 7.24 \\
\hline 68 & 120.56 & 1.79 & 120.17 & 0.56 & 11.58 & 0.68 & -3.09 & 7.00 \\
\hline 70 & 125.22 & 1.35 & 119.53 & 0.48 & 9.73 & 0.69 & 19.33 & 8.80 \\
\hline 71 & 119.57 & 0.77 & 121.24 & 0.25 & 14.79 & 0.21 & 74.83 & 3.68 \\
\hline 74 & 112.49 & 0.82 & 130.36 & 0.32 & 23.82 & 0.11 & 89.45 & 1.03 \\
\hline 75 & 98.14 & 1.83 & 141.30 & 0.91 & 26.37 & 0.12 & 92.63 & 0.87 \\
\hline 76 & 22.97 & 6.45 & 160.63 & 1.17 & 29.49 & 0.05 & 53.85 & 0.24 \\
\hline
\end{tabular}

Table S3. The amplitudes $\sigma_{\gamma}=2 \sigma_{\alpha}=2 \sigma_{\beta}$ of the axially-symmetric 3-D GAF model as well as the angles $C^{\prime} N C^{\alpha}$ and $C^{\prime} N H^{N}$ and the internal correlation time $\tau_{\text {int }}$ determined from the rates of Table S1 using an axially-symmetric 3-D GAF model 